ARTICLE

Received 18 Mar 2015 | Accepted 5 Jun 2015 | Published 22 Jul 2015

DOI: $10.1038 /$ ncomms 8749

OPEN

\title{
Patterned arrays of lateral heterojunctions within monolayer two-dimensional semiconductors
}

Masoud Mahjouri-Samani ${ }^{1}$, Ming-Wei Lin¹, Kai Wang ${ }^{1}$, Andrew R. Lupini², Jaekwang Lee', Leonardo Basile ${ }^{1,3}$, Abdelaziz Boulesbaa1, Christopher M. Rouleau', Alexander A. Puretzky¹, Ilia N. Ivanov', Kai Xiao1, Mina Yoon ${ }^{1} \&$ David B. Geohegan ${ }^{1}$

The formation of semiconductor heterojunctions and their high-density integration are foundations of modern electronics and optoelectronics. To enable two-dimensional crystalline semiconductors as building blocks in next-generation electronics, developing methods to deterministically form lateral heterojunctions is crucial. Here we demonstrate an approach for the formation of lithographically patterned arrays of lateral semiconducting heterojunctions within a single two-dimensional crystal. Electron beam lithography is used to pattern $\mathrm{MoSe}_{2}$ monolayer crystals with $\mathrm{SiO}_{2}$, and the exposed locations are selectively and totally converted to $\mathrm{MoS}_{2}$ using pulsed laser vaporization of sulfur to form $\mathrm{MoSe}_{2} / \mathrm{MoS}_{2}$ heterojunctions in predefined patterns. The junctions and conversion process are studied by Raman and photoluminescence spectroscopy, atomically resolved scanning transmission electron microscopy and device characterization. This demonstration of lateral heterojunction arrays within a monolayer crystal is an essential step for the integration of two-dimensional semiconductor building blocks with different electronic and optoelectronic properties for high-density, ultrathin devices.

\footnotetext{
${ }^{1}$ Center for Nanophase Materials Sciences, Oak Ridge National Laboratory, Oak Ridge, Tennessee 37831, USA. ${ }^{2}$ Materials Science and Technology Division, Oak Ridge National Laboratory, Oak Ridge, Tennessee 37831, USA. ${ }^{3}$ Departamento de Física, Escuela Politécnica Nacional, Quito 170525, Ecuador Correspondence and requests for materials should be addressed to M.M.-S. (email: mahjourisamm@ornl.gov) or to D.B.G. (email: geohegandb@ornl.gov).
} 
T wo-dimensional (2D) layered semiconductors ${ }^{1,2}$, notably the transitional metal dichalcogenide (TMD) families $\left(\mathrm{MX}_{2}: \mathrm{M}=\mathrm{Mo}, \mathrm{W}, \mathrm{Nb} ; \mathrm{X}=\mathrm{S}, \mathrm{Se}, \mathrm{Te}\right)^{1}$ in which $\mathrm{M}$ atoms are sandwiched between two planes of $\mathrm{X}$ atoms, have recently attracted significant attention due to their novel electronic and optoelectronic properties and potential applications for new generations of flexible field-effect transistors ${ }^{3,4}$, photovoltaics ${ }^{5}$, light-emitting diodes $^{6}$ and sensors $^{7}$. High-quality mono- and few-layer samples for prototype devices have been synthesized via various techniques including mechanical cleavage ${ }^{8}$, chemical exfoliation ${ }^{9,10}$, vapour phase transport ${ }^{11,12}$ and laser-based methods ${ }^{13,14}$. However, the next challenge for $2 \mathrm{D}$ material-based ultrathin integrated circuits is the controllable formation of semiconductor heterojunctions, either by vertically stacking different layers or by formation of lateral heterojunctions of different semiconductors within a single layer ${ }^{15-17}$. Lithographic patterning and compatible synthesis approaches are required for high-density integration of semiconductor heterojunctions, as was recently demonstrated for lateral graphene interconnects embedded within an insulating monolayer of boron nitride by etching and regrowth processes ${ }^{17}$. Alternatively, phase engineering of patterned regions of $\mathrm{MoS}_{2}$ crystals by exposure to liquid $n$-butyl lithium has recently achieved the transformation from a semiconducting to a metallic, metastable phase within a single layer ${ }^{4}$.

The most commonly explored method for the formation of a single heterojunction is vapour transport growth (VTG), which has resulted in lateral and vertical epitaxy between semiconducting TMDs such as $\mathrm{MoS}_{2} / \mathrm{MoSe}_{2}, \mathrm{MoSe}_{2} / \mathrm{WSe}_{2}$ and $\mathrm{MoS}_{2} / \mathrm{WS}_{2}$ (refs 18-20). By alternating precursor vapours and adjusting the growth temperature, disparate materials can be nucleated and grown on the edges (lateral junctions) or on top (vertical junctions) of as-grown crystals. However, typical VTG involves well-known challenges of highly non-uniform spatial control over nucleation, growth and layer control, owing to poor understanding and control over gas flow dynamics, chemical reactions and boundary layer diffusion. So far, these challenges as well as contamination by reaction byproducts, and reactivity at exposed crystal edges have limited the scalable and controllable formation of patterned heterojunctions between TMDs by this route.

Here we demonstrate the formation of patterned arrays of lateral heterojunctions between 2D layered semiconductors, $\mathrm{MoSe}_{2} / \mathrm{MoS}_{2}$, within the confines of a single monolayer $\mathrm{MoSe}_{2}$ crystal. Heterojunction arrays are formed by sulfur replacement of Se (refs 21,22) within $\mathrm{MoSe}_{2}$ (lattice constant $3.288 \AA$, optical bandgap $1.55 \mathrm{eV})^{19,23}$, converting it to $\mathrm{MoS}_{2}$ (lattice constant
$3.160 \AA$, optical bandgap $1.85 \mathrm{eV})^{24,25}$ in predefined locations. Using well-developed and scalable photolithography and electron beam lithography processes, a variety of patterns exposed on monolayer $\mathrm{MoSe}_{2}$ crystals are selectively converted to $\mathrm{MoS}_{2}$ by pulsed laser vaporization of sulfur, producing predefined arrays of lateral $\mathrm{MoSe}_{2} / \mathrm{MoS}_{2}$ heterojunctions within a single monolayer. This process provides a unique capability for the digital delivery of a precise amount of sulfur atoms with super-thermal kinetic energies during the conversion process, allowing controllable sulfurization for either alloying or total conversion of $\mathrm{MoSe}_{2}$ to $\mathrm{MoS}_{2}$. Raman and photoluminescence (PL) spectroscopy and mapping confirm the formation of periodic heterojunctions. High-angle annular dark-field atomic resolution scanning transmission electron microscopy (STEM) reveals that the $\mathrm{MoS}_{2}$ is comparable in quality to pristine material, with sharp $(\sim 5 \mathrm{~nm})$ heterojunction boundaries. This controllable and versatile formation of lithographically patterned lateral heterojunction arrays offers the potential for their integration as 2D layer 'building blocks', along with metal/insulator domains, for next-generation electronics.

\section{Results}

Formation of patterned arrays of lateral heterojunctions. Figure 1 schematically illustrates the experimental steps for the formation of periodic lateral heterojunctions. The starting monolayer $\mathrm{MoSe}_{2}$ crystals with lateral sizes ranging from 10 to $100 \mu \mathrm{m}$ were synthesized using a previously reported method ${ }^{11,13}$ (Supplementary Fig. 1; Supplementary Note 1). The crystals were then simply masked by conventional patterning processes, followed by selective conversion of the unmasked $\mathrm{MoSe}_{2}$ to $\mathrm{MoS}_{2}$ by pulsed laser vaporization of sulfur. The masking material $\left.(\sim 50 \mathrm{~nm} \mathrm{SiO})_{2}\right)$ was electron beam evaporated and was found to be quite effective in protecting the areas of the $2 \mathrm{D}$ crystal underneath from sulfurization.

Prior to the patterning process and formation of heterojunction arrays, the conversion of $2 \mathrm{D}$ monolayers was studied at various temperatures for different numbers of sulfur pulses to understand and optimize the process (Supplementary Note 2). PL and Raman spectroscopies were used to investigate the crystal conversion process at various conditions and to reveal their optical properties (Supplementary Fig. 2). We found that $\mathrm{MoSe}_{2}$ nanosheets are totally converted to $\mathrm{MoS}_{2}$ for substrate temperatures above $600{ }^{\circ} \mathrm{C}$ and 300 laser-vaporized sulfur pulses, whereas temperatures and laser pulses below $600^{\circ} \mathrm{C}$ and 300 pulses, respectively, resulted in the formation of intermediate compositions. At temperatures above $800^{\circ} \mathrm{C}$, decomposition and

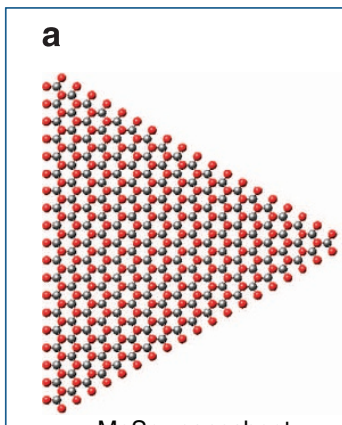

$\mathrm{MoSe}_{2}$ nanosheet

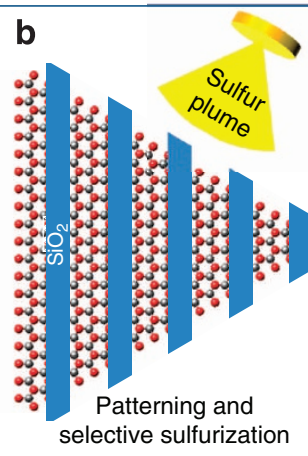

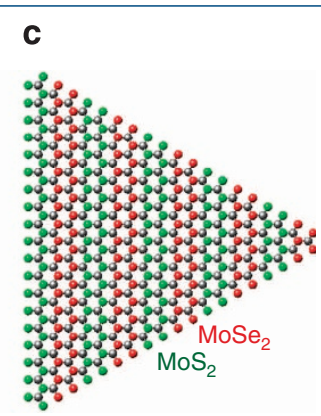

Heterojunction array

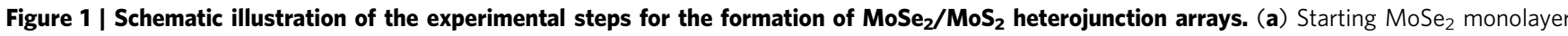
crystal. (b) $\mathrm{MoSe}_{2}$ is patterned by e-beam lithography and $\mathrm{SiO}_{2}$ deposition, followed by sulfurization of uncovered areas. The $\mathrm{SiO}_{2}$ mask is used to prevent the underlying $\mathrm{MoSe}_{2}$ regions from reacting with sulfur while the exposed regions are converted to $\mathrm{MoS}_{2}$. (c) Formation of arrays of lateral $\mathrm{MoSe}_{2} / \mathrm{MoS}_{2}$ heterojunctions within the monolayer crystal. 
damage of the crystals occurred. Therefore, substrate temperatures were fixed at $700{ }^{\circ} \mathrm{C}$ with 400 sulfur pulses to ensure a complete conversion of $\mathrm{MoSe}_{2}$ to $\mathrm{MoS}_{2}$. Conversion of $\mathrm{WSe}_{2}$ to $\mathrm{WS}_{2}$ was also achieved by the same process (Supplementary Fig. 3; Supplementary Note 3) to demonstrate the broad applicability and advantages of the technique.

The thermodynamic stability and optical bandgaps of various $2 \mathrm{D}$ transition metal dichalcogenide alloys $\left(\mathrm{MX}_{2(1-x)} \mathrm{X}_{2 x}^{\prime}\right.$ where $\mathrm{M}=\mathrm{Mo}$, $\mathrm{W}$ and $\mathrm{X}, \mathrm{X}^{\prime}=\mathrm{S}$, Se, Te) have previously been computationally predicted to understand the mixing and phasesegregation behaviour for different alloy compositions ${ }^{26,27}$. Of these, $\mathrm{MoS}_{2 x} \mathrm{Se}_{2(1-x)}$ alloys were found to have the lowest free energy of mixing ${ }^{26,27}$, yet all $\mathrm{MX}_{2(1-x)} \mathrm{X}_{2 x}^{\prime}$ were predicted to form stable alloys with complete miscibility at the moderate temperatures typically employed during chemical vapour deposition or bulk synthesis, and with continuously tunable direct bandgaps, making them good candidates for 2D optoelectronics $^{26}$.

Spectroscopic characteristics of heterojunctions. Raman and PL spectroscopy were used to probe the structures spatially, monitor the conversion degree and to map the heterojunction arrays within the monolayer crystals. Figure 2a,b shows optical and atomic force microscopy images of a typical $\mathrm{MoSe}_{2} 2 \mathrm{D}$ crystal. The corresponding Raman maps of the crystal before and after the full conversion process are shown in Fig. $2 \mathrm{c}$,d. The $\mathrm{MoSe}_{2}$ and $\mathrm{MoS}_{2}$ Raman maps are plotted for the $\mathrm{E}_{2 \mathrm{~g}}^{1}$ mode of $\mathrm{MoSe}_{2}$ at $238 \mathrm{~cm}^{-1}$ and $E_{2 \mathrm{~g}}^{1}$ modes of $\mathrm{MoS}_{2}$ at $403 \mathrm{~cm}^{-1}$, respectively ${ }^{10,13}$. Representative Raman and PL spectra of the flake before and after the conversion process are shown in Fig. 2e,f, indicating $\mathrm{MoSe}_{2}$ and $\mathrm{MoS}_{2}$ Raman peaks similar to those reported in the literature ${ }^{10,13}$. The uniform intensity distribution in the Raman maps indicates spatial uniformity of the $2 \mathrm{D}$ crystals both before and after the conversion. Figure $2 \mathrm{~g}, \mathrm{~h}$ shows the formation of various lateral heterojunction arrays prepared by our patterning and selective conversion process. Similarly, the uniform intensity of Raman maps clearly indicates the spatial uniformity of the $\mathrm{MoSe}_{2}$ and $\mathrm{MoS}_{2}$ domains, and formation of heterojunction arrays within the monolayer crystals. The Raman and PL spectra obtained from the pristine and converted regions of the crystals are similar to the ones shown in Fig. 2e,f.

STEM characterization of heterojunctions. Crystalline structures of the converted and pristine regions as well as their heterojunction boundaries were also studied by atomic resolution Z-contrast STEM. Figure 3a,b shows the optical image and corresponding Raman map of a typical patterned/converted layer transferred onto a grid for STEM imaging (Supplementary Fig. 4; Supplementary Note 4). As shown in Fig. 3c, atomic resolution Z-contrast STEM images are taken at the heterojunction that clearly shows both $\mathrm{MoSe}_{2}$ (Fig. 3d) and $\mathrm{MoS}_{2}$ (Fig. 3e) 2D crystal domains. The line and surface intensity profiles of the selected regions in Fig. 3c are shown in Fig. 3f,g. Likewise, Fig. 3h,i shows a STEM image of a boundary with its corresponding electron energy loss spectroscopy map showing the sulfur content (in green) as a function of position. It is clear that both pristine $\mathrm{MoSe}_{2}$ and converted $\mathrm{MoS}_{2}$ regions lie within the same honeycomb lattice with no grain boundaries-that is, the $\mathrm{MoSe}_{2}$ crystal serves as a template, maintaining the same crystal orientations throughout the whole structure. As can be seen from the STEM image, the interface has a finite width similar to that reported for
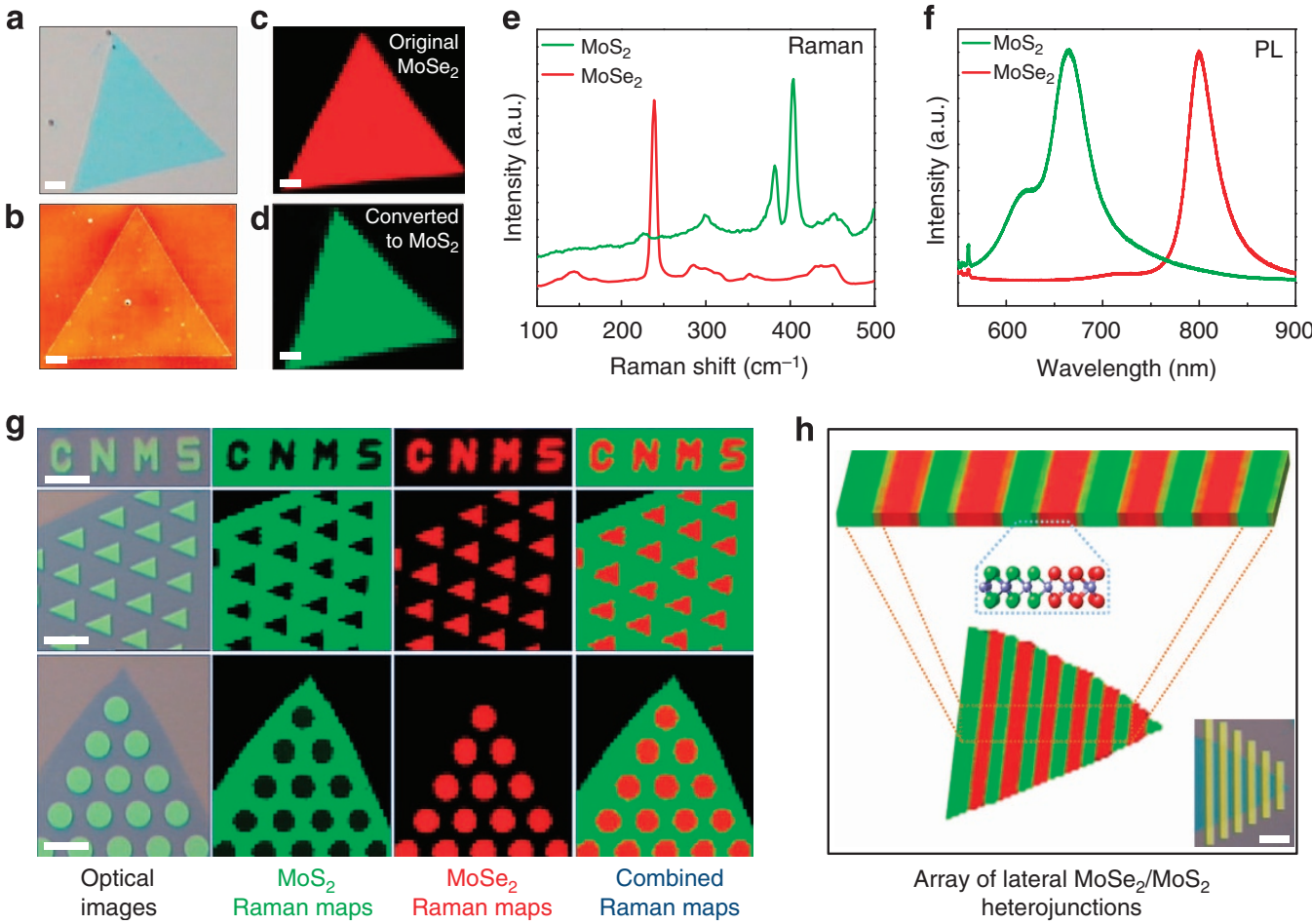

Figure 2 | Conversion of $\mathbf{M o S e}_{\mathbf{2}}$ to $\mathbf{M o S}_{\mathbf{2}}$ and the formation of lateral heterojunction arrays. (a,b) Optical and atomic force microscopy images of a typical $\mathrm{MoSe}_{2}$ monolayer with a lateral size of $\sim 40 \mu \mathrm{m}$. (c,d) Raman maps of a monolayer nanosheet before and after the complete conversion process (400 pulses at $700^{\circ} \mathrm{C}$ ), respectively, indicating uniform intensity across the entire crystal. (e,f) Representative Raman and PL spectra of the pristine MoSe $e_{2}$ and converted $\mathrm{MoS}_{2}$ regions. ( $\left.\mathbf{g}, \mathbf{h}\right)$ Various examples of lateral heterojunction arrays formed within monolayer crystals by patterning and selective conversion processes. The green, red and combined Raman maps are obtained from corresponding optical images, representing the $\mathrm{MoS}_{2}$ (intensity map at $403 \mathrm{~cm}^{-1}$ ), $\mathrm{MoSe}_{2}$ (intensity map at $238 \mathrm{~cm}^{-1}$ ) and overlaid $\mathrm{MoSe}_{2} / \mathrm{MoS}_{2}$ regions, respectively. Scale bars, $5 \mu \mathrm{m}$. 


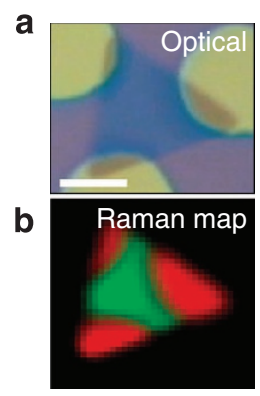

h
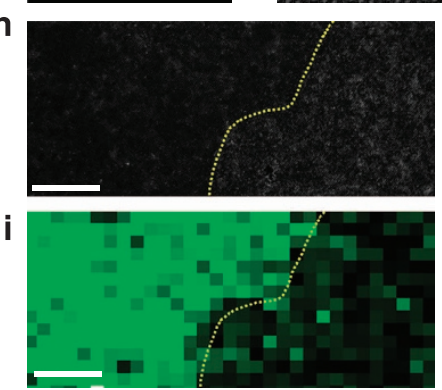
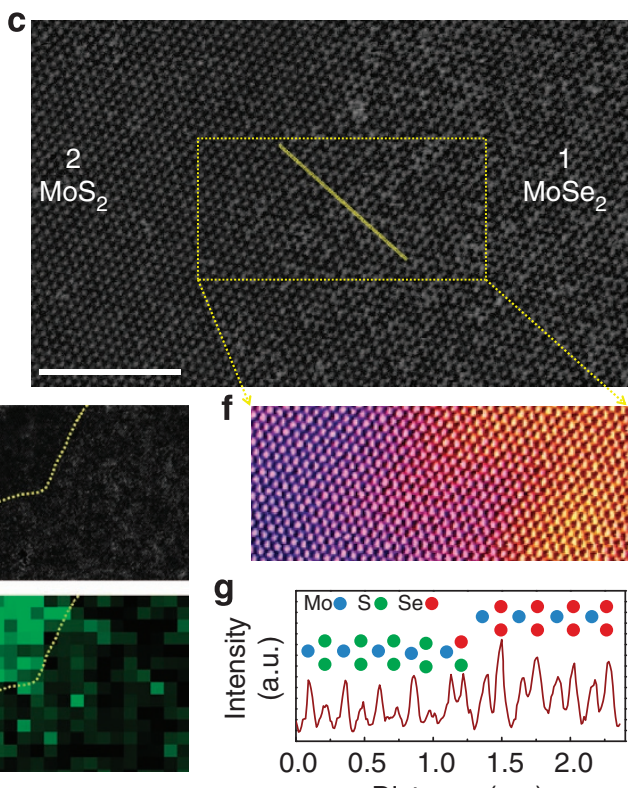
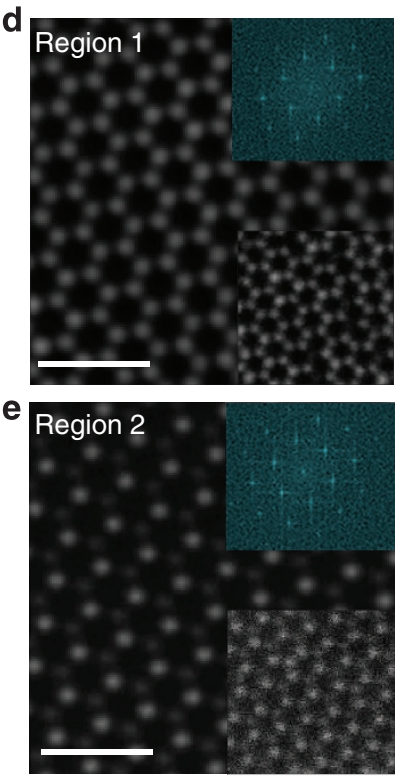

Figure 3 | STEM Z-contrast image and elemental imaging of a heterojunction. (a,b) Optical image and corresponding Raman map of a patterned nanosheet on a $\mathrm{SiO}_{2}$ substrate (scale bar, $5 \mu \mathrm{m}$ ). The $\mathrm{SiO}_{2}$ masks (circular discs in a) are removed during $\mathrm{KOH}$ etching, and the transfer of the nanosheet onto the TEM grids. (c) Low-magnification Z-contrast image of the nanosheet showing the $\mathrm{MoSe}_{2}$ and $\mathrm{MoS}_{2}$ regions with a finite boundary across the domains (scale bars, $5 \mathrm{~nm}$ ). (d,e) Fourier filtered images of the atomic resolution Z-contrast images of the $\mathrm{MoSe}_{2}$ and $\mathrm{MoS}_{2}$ (bottom insets in the images) domains with corresponding fast Fourier transform patterns (top insets in the images). (f,g) Surface and line intensity profiles of the squared and line-marked regions in $\mathbf{c}$. (h,i) Low-magnification image of a boundary with its corresponding electron energy loss spectroscopy map showing the sulfur concentration (scale bars, $5 \mathrm{~nm}$ ).
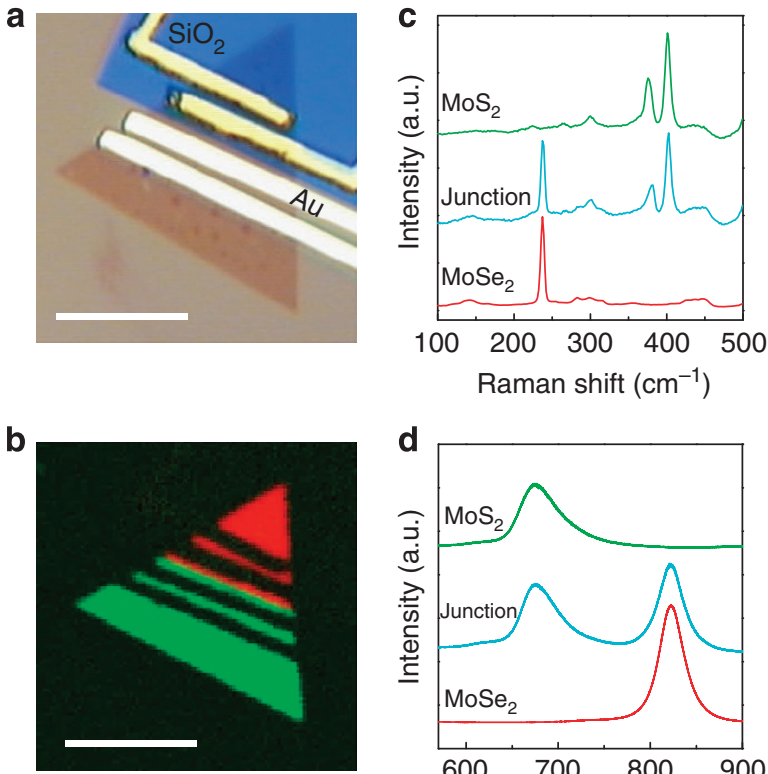

e
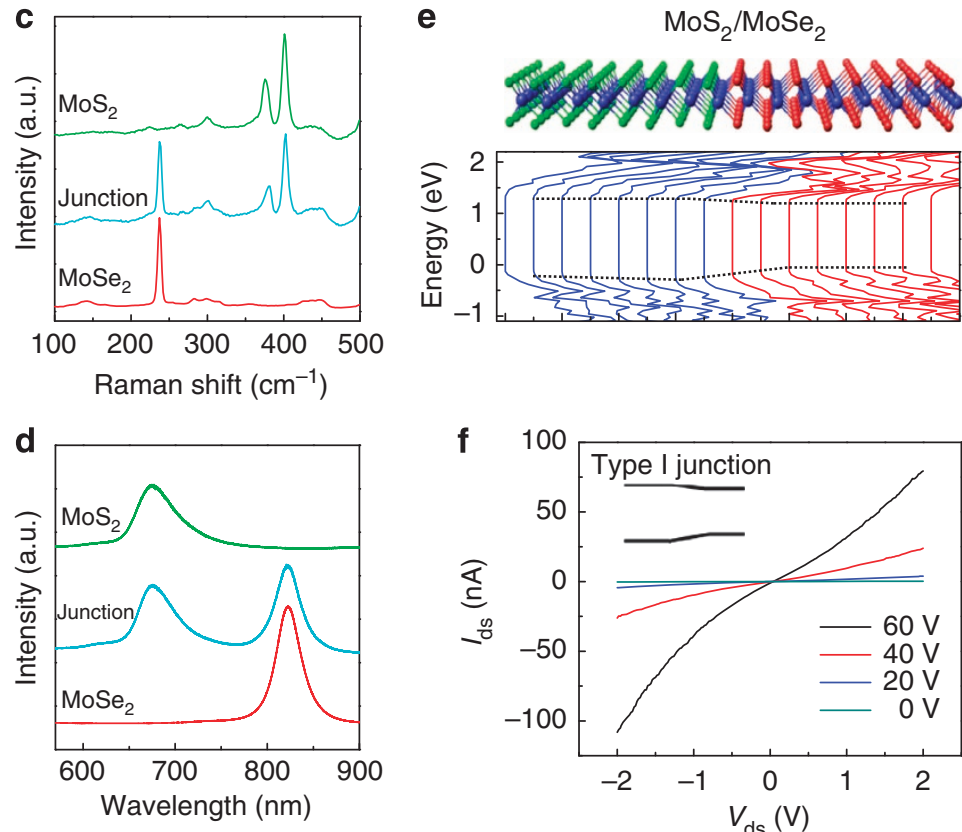

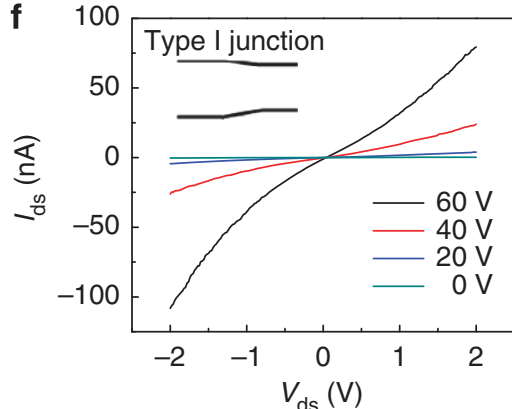

Figure 4 | Electrical transport properties. (a,b) Optical and Raman map of a MoS $2 / \mathrm{MoSe}_{2}$ (green/red) heterojunction device. (c,d) Raman and PL spectra of $\mathrm{MoS}_{2}$ and $\mathrm{MoSe}_{2}$ obtained from each region showing a distinct peak for regions away from the junction and appearance of both peaks at the junction. (e) First-principles DFT calculation of $\mathrm{MoSe}_{2}$ and $\mathrm{MoS}_{2}$ superlattices showing the formation of type-I band alignment. (f) $I_{\mathrm{ds}}-V_{\mathrm{ds}}$ characteristics of the heterojunction showing an $n-n$ junction behaviour at the interface.

$\mathrm{MoSe}_{2} / \mathrm{WSe}_{2}$ heterojunctions ${ }^{19}$. The boundary appears to be a $\mathrm{MoS}_{x} \mathrm{Se}_{1-x}$ ternary alloy with a composition gradient over a distance of several nanometres. The sharpness of the heterojunctions is related to the e-beam lithography and patterning processes used in this work, which can be improved further.
Electrical transport properties. The electrical transport properties of pristine $\mathrm{MoSe}_{2}$, converted $\mathrm{MoS}_{2}$ and their heterojunctions were studied by forming a device structure. Figure 4a shows an example of a device structure fabricated in this work. Prior to the conversion process, two electrodes were fabricated on one side of 
the crystal to measure the transport properties of the pristine $\mathrm{MoSe}_{2}$. Then, this part was covered with a $\mathrm{SiO}_{2}$ mask and the sulfurization was carried out, resulting in the conversion of the exposed half of the crystal to $\mathrm{MoS}_{2}$. Two electrodes were then fabricated on this half to measure the electrical transport characteristics of the converted $\mathrm{MoS}_{2}$ region. Figure $4 \mathrm{~b}$ shows the Raman map of the device with red and green colours clearly indicating the $\mathrm{MoSe}_{2}$ and $\mathrm{MoS}_{2}$ regions, respectively, and their boundaries. Representative Raman and PL spectra of these regions are also shown in Fig. 4c,d, indicating distinct Raman and PL peaks for each domain while observing simultaneous $\mathrm{MoSe}_{2}$ and $\mathrm{MoS}_{2}$ peaks right at the junction.

To understand band alignment at the interface, first-principles calculations, based on density functional theory, were performed using the Vienna ab initio Simulation Package (Supplementary Note 5). As illustrated in Fig. 4e, we considered a supercell in which eight zigzag rows of $\mathrm{MoS}_{2}$ joined eight rows of $\mathrm{MoSe}_{2}$, and plotted the partial density of states projected onto the atomic rows in the $\mathrm{MoS}_{2}$ and $\mathrm{MoSe}_{2}$ across the heterojunction. From the projected density of states, we can see that there exists an obvious graded bandgap across the heterojunction. In particular, we find that the lateral $\mathrm{MoS}_{2} / \mathrm{MoSe}_{2}$ heterojunction forms a type-I band alignment, as indicated by the dotted line (Fig. 4e), since the bandgap of $\mathrm{MoS}_{2}$ entirely overlaps that of $\mathrm{MoSe}_{2}$.

$I_{\mathrm{ds}}-V_{\mathrm{ds}}$ curves were obtained from pristine $\mathrm{MoSe}_{2}$, converted $\mathrm{MoS}_{2}$ and their heterojunction to investigate their transport characteristics. According to the measured $I_{\mathrm{ds}}-V_{\mathrm{ds}}$ curves, both $\mathrm{MoSe}_{2}$ and converted $\mathrm{MoS}_{2}$ show n-type behaviour (Supplementary Fig. 5) similar to the previously reported work $^{11,12}$. Consequently, as shown in Fig. 4f, their boundary shows transport characteristic of an $\mathrm{n}-\mathrm{n}$ heterojunction. The smaller current amplitude across the heterojunction boundary may be attributed to electron scattering at the heterojunction and the type of their band alignment.

\section{Discussion}

In conclusion, through lithographic patterning and controllable conversion of $\mathrm{MoSe}_{2}$ to $\mathrm{MoS}_{2}$, arrays of lateral $\mathrm{MoSe}_{2} / \mathrm{MoS}_{2}$ heterojunctions have been synthesized within a monolayer $2 \mathrm{D}$ crystal. Electron beam lithography coupled with vacuum deposition of $\mathrm{SiO}_{2}$ masks and sulfur, two scalable techniques used in the semiconductor industry, were employed to form sharp $(\sim 5 \mathrm{~nm})$ heterojunctions comparable to those formed by heteroepitaxy, without the non-uniformities typically introduced in VTG processes. Pulsed laser vaporization provided a controllable and rapid method to explore the conversion dynamics through the digital delivery of precise quantities of the sulfur precursor. The patterning and selective conversion process demonstrated here for semiconductor heterojunctions appears to be a powerful technique that could be extended to form other metallic, insulating and semiconducting regions within $2 \mathrm{D}$ materials required for ultrathin electronics.

\footnotetext{
Methods

E-beam patterning process. Electron beam lithography (FEI DB-FIB with Raith pattern writing software) was used for $\mathrm{MoSe}_{2} / \mathrm{MoS}_{2}$ heterostructure device fabrication. First, a layer of PMMA 495A4 was spin-coated on top of the $\mathrm{MoSe}_{2}$ flakes, followed by a $180^{\circ} \mathrm{C}$ bake on a hot plate. After pattern writing and developing, $50 \mathrm{~nm}$ of $\mathrm{SiO}_{2}$ was deposited using electron beam evaporation to serve as conversion masks. For device fabrication, $5 \mathrm{~nm}$ of Ti and $30 \mathrm{~nm}$ of Au were deposited using electron beam evaporation to serve as electrodes on the $\mathrm{MoSe}_{2}$ flakes. After the $\mathrm{MoSe}_{2}$ was converted to $\mathrm{MoS}_{2}$, electron beam lithography was conducted again to place electrodes on the $\mathrm{MoS}_{2}$. Finally, lift-off using acetone/IPA was used to reveal well-defined electrodes on both the $\mathrm{MoSe}_{2}$ and converted $\mathrm{MoS}_{2}$ flakes.
}

Conversion process. The conversion was performed in a vacuum $\left(\sim 10^{-5}\right.$ Torr $)$ chamber where a forward directed laser-vaporized sulfur plume was generated and digitally delivered to the surface of the crystals ${ }^{28}$. The vaporization target was prepared by compressing sulfur powder ( $99 \%$, Sigma-Aldrich) into a pellet, and the laser fluence was adjusted to about $0.5 \mathrm{~J} \mathrm{~cm}^{-2}$ (spot size of $2 \times 5 \mathrm{~mm}$ and repetition rate of $0.5 \mathrm{~Hz}$ ) to ensure the transfer of a fixed amount of sulfur onto the crystals for all of the experimental conditions. The conversion was conducted under various substrate temperatures and laser pulses to understand and optimize the process (Supplementary Fig. 2; Supplementary Note 2).

Optical characterizations. Raman and PL mapping and spectroscopy were performed in a Renishaw inVia micro-Raman system using a 532-nm laser excitation source, $1,800 \mathrm{~g} \mathrm{~mm}^{-2}$ grating and a laser power of about $1-5 \mathrm{~mW}$ through $\times 50$ and $\times 100$ objective lenses at room temperature. Maps were obtained with step sizes ranging from 0.3 to $1 \mu \mathrm{m}$. WiRE software was used to analyse and plot the maps for individual $\mathrm{MoSe}_{2} / \mathrm{MoS}_{2}$ sections and their overlaid images.

STEM Z-contrast imaging and analysis. All STEM samples were baked at $160^{\circ} \mathrm{C}$ for $8 \mathrm{~h}$ under vacuum before the microscopy experiment. STEM imaging was performed on an aberration-corrected Nion UltraSTEM-100 operating at $60 \mathrm{kV}$. The convergence semi-angle for the incident probe was $31 \mathrm{mrad}$. Z-contrast images were gathered for a half-angle range of $\sim 86-200 \mathrm{mrad}$. Electron energy loss spectroscopy and spectrum imaging was performed using a Gatan Enfina with a nominal collection angle of $35 \mathrm{mrad}$.

Electrical transport characterization. The electrical measurement of $\mathrm{MoSe}_{2} / \mathrm{MoS}_{2}$ field-effect transistor devices was conducted in a vacuum chamber $\left(\sim 10^{-6}\right.$ Torr) using a Keithley 4200 semiconductor analyzer. The drain/source voltage $\left(V_{\mathrm{ds}}\right)$ was set at $2 \mathrm{~V}$ with a gate voltage that swept from -60 to $+60 \mathrm{~V}$ for transport measurement.

\section{References}

1. Chhowalla, M. et al. The chemistry of two-dimensional layered transition metal dichalcogenide nanosheets. Nat. Chem. 5, 263-275 (2013).

2. Butler, S. Z. et al. Progress, challenges, and opportunities in two-dimensional materials beyond graphene. ACS Nano 7, 2898-2926 (2013).

3. Pradhan, N. R. et al. Ambipolar molybdenum diselenide field-effect transistors field-effect and hall mobilities. ACS Nano 8, 7923-7929 (2014).

4. Kappera, R. et al. Phase-engineered low-resistance contacts for ultrathin $\mathrm{MoS}_{2}$ transistors. Nat. Mater. 13, 1128-1134 (2014).

5. Tsai, M. L. et al. Monolayer $\mathrm{MoS}_{2}$ heterojunction solar cells. ACS Nano 8, 8317-8322 (2014)

6. Sundaram, R. S. et al. Electroluminescence in single layer $\mathrm{MoS}_{2}$. Nano Lett. 13, 1416-1421 (2013).

7. Perkins, F. K. et al. Chemical vapor sensing with monolayer $\mathrm{MoS}_{2}$. Nano Lett. 13, 668-673 (2013)

8. Li, H., Wu, J. M. T., Yin, Z. Y. \& Zhang, H. Preparation and applications of mechanically exfoliated single-layer and multi layer $\mathrm{MoS}_{2}$ and $\mathrm{WSe}_{2}$ nanosheets. Acc. Chem. Res. 47, 1067-1075 (2014).

9. Coleman, J. N. et al. Two-dimensional nanosheets produced by liquid exfoliation of layered materials. Science 331, 568-571 (2011).

10. Zheng, J. et al. High yield exfoliation of two-dimensional chalcogenides using sodium naphthalenide. Nat. Commun. 5, 2995 (2014).

11. Wang, X. et al. Chemical vapor deposition growth of crystalline monolayer $\mathrm{MoSe}_{2}$. ACS Nano 8, 5125-5131 (2014).

12. Najmaei, S. et al. Vapour phase growth and grain boundary structure of molybdenum disulphide atomic layers. Nat. Mater. 12, 754-759 (2013).

13. Mahjouri-Samani, M. et al. Digital transfer growth of patterned 2D metal chalcogenides by confined nanoparticle evaporation. ACS Nano $\mathbf{8}$, 11567-11575 (2014).

14. Mahjouri-Samani, M. et al. Pulsed laser deposition of photoresponsive two-dimensional GaSe nanosheet networks. Adv. Funct. Mater. 24, 6365-6371 (2014).

15. Zeng, Q. et al. Band engineering for novel two-dimensional atomic layers. Small 11, 1868-1884 (2014).

16. Duesberg, G. S. Heterojunctions in $2 \mathrm{~d}$ semiconductors a perfect match. Nat. Mater. 13, 1075-1076 (2014).

17. Levendorf, M. P. et al. Graphene and boron nitride lateral heterostructures for atomically thin circuitry. Nature 488, 627-632 (2012).

18. Duan, X. et al. Lateral epitaxial growth of two-dimensional layered semiconductor heterojunctions. Nat. Nanotechnol. 9, 1024-1030 (2014).

19. Huang, C. et al. Lateral heterojunctions within monolayer $\mathrm{MoSe}_{2}-\mathrm{WSe}_{2}$ semiconductors. Nat. Mater. 13, 1096-1101 (2014).

20. Gong, Y. et al. Vertical and in-plane heterostructures from $\mathrm{WS}_{2} / \mathrm{MoS}_{2}$ monolayers. Nat. Mater. 13, 1135-1142 (2014).

21. Su, S.-H. et al. Controllable synthesis of band gap-tunable and monolayer transition metal dichalcogenide alloys. Front. Energy Res. 2, 1-5 (2014). 
22. Li, H. et al. Growth of alloy $\operatorname{MoS}_{(2 x)} \mathrm{Se}_{2(1-x)}$ nanosheets with fully tunable chemical compositions and optical properties. J. Am. Chem. Soc. 136, 3756-3759 (2014).

23. James, P. B. \& Lavik, M. T. Crystal structure of $\mathrm{MoSe}_{2}$. Acta Crystallogr. 16, $1183(1963)$

24. Young, P. A. Lattice parameter measurements on molybdenum disulphide. J. Phys. D Appl. Phys. 1, 936 (1968).

25. Nayak, A. P. et al. Pressure-dependent optical and vibrational properties of monolayer molybdenum disulfide. Nano Lett. 15, 346-353 (2015).

26. Kang, J., Tongay, S., Li, J. B. \& Wu, J. Q. Monolayer semiconducting transition metal dichalcogenide alloys: stability and band bowing. J. Appl. Phys. 113, 143703 (2013).

27. Komsa, H. P. \& Krasheninnikov, A. V. Two-dimensional transition metal dichalcogenide alloys: stability and electronic properties. J. Phys. Chem. Lett. 3, 3652-3656 (2012)

28. Lowndes, D. H., Geohegan, D. B., Puretzky, A. A., Norton, D. P. \& Rouleau, C. M. Synthesis of novel thin-film materials by pulsed laser deposition. Science 273, 898-903 (1996).

\section{Acknowledgements}

Synthesis science including crystal growth, in situ plume diagnostics, TEM analysis, SEM and AFM studies and conversion technique development (M.M.-S., K.W., J.L., A.R.L., K.X., D.B.G., C.M.R., A.A.P. and M.Y.) was supported by the US Department of Energy, Office of Science, Basic Energy Sciences, Materials Sciences and Engineering Division and performed in part as a user project at the Center for Nanophase Materials Sciences, which is a DOE Office of the Science User Facility. Characterization science at Center for Nanophase Materials Sciences (CNMS) including optical characterization and lithography techniques (M.-W.L., A.B. and I.N.I.) was supported by the Scientific User Facilities Division. L.B. was supported by the National Secretariat of Higher Education, Science, Technology and Innovation of Ecuador (SENESCYT).

\section{Author contributions}

M.M.-S. designed and performed the synthesis and conversion experiments, and obtained Raman and PL data with I.N.I.. M.-W.L. and K.X. participated in patterning, device fabrication and transport characterization. J.L. and M.Y. performed theoretical modelling. K.W. participated in the synthesis of $\mathrm{MoSe}_{2}$ nanosheets. A.R.L. and L.B. carried out the STEM study and analysis. C.M.R., A.B., A.A.P. and D.B.G. participated in design study, data analysis, discussions and manuscript preparation. All the authors discussed the results and commented on the manuscript.

\section{Additional information}

Supplementary Information accompanies this paper at http://www.nature.com/ naturecommunications

Competing financial interests: The authors declare no competing financial interests.

Reprints and permission information is available online at http://npg.nature.com/ reprintsandpermissions/

How to cite this article: Mahjouri-Samani, M. et al. Patterned arrays of lateral heterojunctions within monolayer two-dimensional semiconductors. Nat. Commun. 6:7749 doi: 10.1038/ncomms8749 (2015).

\section{(c) (i)}

This work is licensed under a Creative Commons Attribution 4.0 International License. The images or other third party material in this article are included in the article's Creative Commons license, unless indicated otherwise in the credit line; if the material is not included under the Creative Commons license, users will need to obtain permission from the license holder to reproduce the material. To view a copy of this license, visit http://creativecommons.org/licenses/by/4.0/ 